\title{
Efeitos Adversos durante a Infusão de Infliximabe em Crianças e Adolescentes - Estudo Multicêntrico
}

\section{Adverse Reactions during Infliximab Infusion In Children and Adolescents - Multicenter Study}

\author{
Cássia Maria Passarelli Lupoli Barbosa ${ }^{(1)}$, Maria Teresa R. A. Terreri( ${ }^{(2)}$, Sheila K. F. Oliveira ${ }^{(3)}$, \\ Marta Cristine Felix Rodrigues ${ }^{(4)}$, Blanca Elena Rios Gomes Bica ${ }^{(5)}$, Silvana Sacchetti ${ }^{(6)}$, \\ Paulo Roberto Stocco Romanelli( ${ }^{(7)}$, Cláudio Arnaldo Len ${ }^{(8)}$, Maria Odete Esteves Hilário ${ }^{(9)}$
}

\section{RESUMO}

Objetivo: Avaliar a freqüência e a gravidade dos efeitos adversos durante a infusão de infliximabe. Material e Métodos: Avaliação retrospectiva de prontuários de 58 pacientes acompanhados em cinco serviços de reumatologia pediátrica. Todos apresentavam doença refratária ou eram intolerantes ao uso de uma ou mais drogas modificadoras de doença e receberam uma ou mais infusões de infliximabe. A análise estatística foi descritiva, levando-se em conta a freqüência e o tipo dos efeitos adversos, assim como sua relação com o número de infusões, a dose de infliximabe e a indicação. Resultados: A média da idade na época da introdução do infliximabe foi de 12 anos e 9 meses e a média do tempo de evolução da doença foi de 7 anos e 7 meses. A principal indicação da medicação foi artrite idiopática juvenil (43). Dos 58 pacientes avaliados, $25(43,1 \%)$ apresentaram efeitos adversos durante a infusão e em 17 (29,3\%), a medicação teve de ser suspensa. As reações que ocorreram foram: dispnéia (dez), náuseas e vômitos (oito), rash cutâneo (sete), choque anafilático (seis), rubor facial (cinco), angioedema (quatro), dor torácica (quatro), urticária e hipertensão, entre outras. A reação anafilática foi mais freqüente entre a quarta e a sexta infusão. Conclusão: A freqüência e a gravidade dos efeitos adversos ao infliximabe devem ser levadas em conta, não apenas por ocasião da sua indicação, mas principalmente por ocasião da escolha do local para a sua aplicação, que deve contar com estrutura para atendimento de urgência.

Palavras-chave: anti-TNF, efeitos adversos, reações infusionais, infliximabe, crianças.

\begin{abstract}
Objective: To evaluate the frequency and the severity of the adverse reactions during the infusion of infliximab. Methods: We performed a retrospective chart review of 58 patients, followed up at 5 paediatric rheumatology centers. All patients presented refractory disease or were intolerant to one or more disease modifying drugs and received one or more infliximab infusions. The data analysis was descriptive, considering the frequency and the kind of adverse reactions as well as the number of the infusions, the infliximab dose and the indication. Results: The mean age at the onset of infliximab was 12 years and 9 months and the mean of the disease duration was 7 years and 7 months. The main indication of the drug was juvenile idiopathic arthritis (43). Twenty five out of 58 patients (43.1\%) presented adverse reactions during the infusion and 17 (29,3\%) had to withdraw the treatment. The reactions were: dyspnea (ten), nausea and vomiting (eight), rash (seven), anaphylaxis (six), flush (five), angioedema (four), chest pain (four) besides urticaria, hypertension, among others. The anaphylaxis was more frequent between the fourth and the sixth infusion. Conclusion: The frequency and the severity of adverse reactions to infliximab must be considered not only in relation to its indication, but mainly for the choice of the center to the drug infusion where emergency treatment may be performed.
\end{abstract}

Keywords: anti-TNF, adverse reactions, infusion reactions, infliximab, children.

\footnotetext{
Recebido em 19/2/2008. Aprovado, após revisão, em 23/6/2008. Declaramos a inexistência de conflitos de interesse

1. Médica pediatra do Setor de Reumatologia Pediátrica do Departamento de Pediatria da Universidade Federal de São Paulo (Unifesp)

2. Professora afiliada do Setor de Reumatologia Pediátrica do Departamento de Pediatria da Unifesp.

3. Professora-associada de Pediatria da Faculdade de Medicina da Universidade Federal do Rio de Janeiro (UFRJ), Chefe do Serviço de Reumatologia Pediátrica do Instituto de Puericultura e Pediatria Martagão Gesteira/UFRJ

4. Médica do Serviço de Reumatologia Pediátrica do Instituto de Puericultura e Pediatria Martagão Gesteira/UFRJ

5. Professora adjunta de Reumatologia Pediátrica da Faculdade de Medicina da UFRJ, Coordenadora do setor de Reumatologia do Adolescente.

6. Médica responsável pelo Serviço de Reumatologia Pediátrica do Departamento de Pediatria da Irmandade da Santa Casa de Misericórdia de São Paulo.

7. Médico especialista em Reumatologia Geral e Pediátrica, assistente técnico da diretoria do Hospital Infantil Menino Jesus (SMS/PMSP).

8. Professor associado do Setor de Reumatologia Pediátrica do Departamento de Pediatria da Unifesp.

9. Professora adjunta. Chefe do setor de Reumatologia Pediátrica do Departamento de Pediatria da Unifesp.

Endereço para correspondência: Cássia M. P. L. Barbosa, Rua Itagyba Santiago, 360, apto. 63, 04635-051, São Paulo, SP, E-mail: cassiapassarelli@uol.com.br
} 


\section{INTRODUÇÃO}

O fator de necrose tumoral alfa (TNF- $\alpha$ ) é uma citocina inflamatória que desempenha papel-chave na resposta imune, na defesa contra microrganismos e no processo inflamatório. Níveis elevados desta citocina foram encontrados em pacientes com artrite idiopática juvenil (AIJ) e com outras doenças do colágeno ${ }^{(1-3)}$.

Atualmente, estão disponíveis três agentes biológicos com ação anti-TNF- $\alpha$ : etanercepte, uma proteína de fusão que é receptor solúvel; infliximabe que é um anticorpo monoclonal IgGl, quimérico humano/murino; e adalimumabe, um anticorpo monoclonal humanizado ${ }^{(4)}$. Todos eles podem ser eficazes no controle da inflamação e na inibição da progressão do dano articular, no controle da uveíte e da doença inflamatória intestinal, mesmo quando a resposta a uma ou mais drogas modificadoras de doença se mostra ineficiente ${ }^{(5-8)}$.

Os efeitos adversos dos bloqueadores do TNF- $\alpha$ incluem infecções, eventos auto-imunes e alérgicos ${ }^{(1,9-13)}$.

Uma vez que o infliximabe é constituído também por uma proteína murina, os efeitos adversos no período de infusão são freqüentes, incluindo a reação anafilática ${ }^{(9,10)}$.

O objetivo deste trabalho foi avaliar a freqüência e a gravidade dos efeitos adversos em pacientes submetidos ao tratamento com infliximabe.

\section{MATERIAL E MÉTODOS}

Foram avaliados, retrospectivamente, os prontuários de 58 pacientes acompanhados em cinco serviços de reumatologia pediátrica, com doença refratária a uma ou mais drogas modificadoras de doença e que receberam uma ou mais infusões de infliximabe, no período de janeiro de 2003 a junho de 2007.

A triagem para tuberculose foi realizada em todos os pacientes por meio de radiografia de tórax e teste tuberculínico (PPD).

Previamente à infusão, os responsáveis foram orientados quanto aos riscos da medicação e assinaram o termo de consentimento livre e esclarecido para sua aplicação e para a publicação dos dados.

A medicação foi preparada e infundida de acordo com as instruções do fabricante: uso de equipo de infusão adaptado a um filtro estéril, não-pirogênico, com baixa ligação a proteínas (tamanho do poro: $1,2 \mu \mathrm{m}$ ou menos), diluição da medicação em água destilada 1:10 e, em seguida em 250 $\mathrm{mL}$ de soro fisiológico $0,9 \%$.
A dose utilizada foi de 3 a $6 \mathrm{mg} / \mathrm{kg} /$ dose (máximo de $300 \mathrm{mg}$ ), dada em uma primeira aplicação, 15 dias e 6 semanas após e, posteriormente, de 8 em 8 semanas, ou eventualmente com intervalos mais curtos, dependendo da evolução do paciente. Apenas uma paciente recebeu dose de $10 \mathrm{mg} / \mathrm{kg}(500 \mathrm{mg})$.

A administração da medicação endovenosa teve duração de pelo menos 2 horas e foi realizada em local equipado para atendimento de urgência, sob cuidados de pessoal habilitado.

$\mathrm{O}$ uso de medicações previamente à infusão de infliximabe variou de um centro para outro, porém todos os pacientes receberam corticoesteróide endovenoso, antihistamínico e paracetamol.

Foram considerados efeitos adversos aqueles que ocorreram durante a infusão ou até uma hora após o seu término. Os pacientes foram prontamente atendidos e evoluíram bem, com regressão completa destes efeitos.

A análise estatística foi descritiva, levando-se em conta a freqüência e o tipo de efeitos adversos, assim como a sua relação com o número de infusões, uso de pré-medicação e dose de infliximabe.

\section{RESULTADOS}

Cinqüenta e oito pacientes foram submetidos a 511 infusões de infliximabe, com média de 8,8 , variando de 1 a 24 infusões por paciente.

A média de idade na época da introdução do infliximabe foi de 12 anos e 9 meses (3-19 anos) e a média do tempo de evolução da doença foi de 7 anos e 7 meses ( 6 meses- 17 anos). A indicação mais freqüente foi AIJ (Tabela 1$)$.

\section{TABela 1}

Diagnóstico de indicação de TERAPIA COM INFLIXIMABE $(\mathrm{N}=\mathbf{5 8})$

\begin{tabular}{lc}
\hline Diagnóstico & Infliximabe (n) \\
\hline Artrite idiopática juvenil & 23 \\
$\quad$ Poliarticular - fator reumatóide negativo & 6 \\
$\quad$ Sistêmica & 5 \\
$\quad$ Artrite relacionada à entesite & 4 \\
$\quad$ Oligoarticular & 4 \\
$\quad$ Poliarticular - fator reumatóide positivo & 1 \\
$\quad$ Psoriásica & 4 \\
Dermatomiosite & 3 \\
Doença inflamatória intestinal & 3 \\
\hline Policondrite & 2 \\
Síndrome de Blau & 1 \\
Síndrome periódica associada ao receptor de TNF (TRAPS) & 1 \\
Síndrome infantil crônica articular neurológica e cutânea & 1 \\
\hline (CINCA) & \\
\hline Vasculite & \\
\hline
\end{tabular}


Dos 58 pacientes, $25(43,1 \%)$ apresentaram efeitos adversos durante a infusão e, em 17 (29,3\%), a medicação foi suspensa. Os efeitos adversos variaram de reações leves a choque anafilático. As reações que ocorreram foram: dispnéia (dez), náuseas e vômitos (oito), rash cutâneo (sete), choque anafilático (seis), rubor facial (cinco), angioedema (quatro), dor torácica (quatro), além de urticária, hipertensão, hipotensão, tosse, sensação de morte, taquicardia e cianose (Tabela 2).

Três pacientes apresentavam história de alergia a outros medicamentos, porquanto um deles manifestou anafilaxia ao infliximabe.

A reação anafilática foi mais freqüente entre a quarta e a sexta dose, embora em um caso tenha ocorrido na primeira e, em outro, na décima oitava aplicação.
Os efeitos adversos foram prontamente diagnosticados, tratados adequadamente e os pacientes evoluíram bem.

\section{DISCUSSÃO}

Os estudos controlados sobre o uso do infliximabe são escassos, e este é o primeiro estudo multicêntrico na faixa etária pediátrica no Brasil.

Dos biológicos disponíveis no Brasil, o infliximabe foi o primeiro a chegar à rede pública e, conseqüentemente, o que vinha sendo utilizado com maior freqüência. Constitui, atualmente, uma alternativa para o tratamento de doenças inflamatórias refratárias ao tratamento, quando há ausência de resposta a uma ou mais drogas modificadoras de doença ${ }^{(5-8,11)}$.

TABELA 2

INDICAÇÕES, EFEITOS ADVERSOS, DOSE E NÚMERO DA INFUSÃo NA REAÇ̃̃o AO INFLIXIMABE (N = 25)

\begin{tabular}{|c|c|c|c|c|c|}
\hline Paciente & Indicação & Efeitos adversos & Dose $\mathrm{mg} / \mathrm{kg}$ & Número da infusão & Suspensão \\
\hline 1 & AIJ oligo/uveíte & Anafilaxia & 3 & 18 & Sim \\
\hline 2 & AIJ oligoarticular & Anafilaxia & 5 & 6 & Sim \\
\hline 3 & EAJ & Dor torácica, dispnéia, broncoespasmo & 5 & 3 & Sim \\
\hline 4 & TRAPS & Anafilaxia & 5 & 4 & Sim \\
\hline 5 & AIJ poliarticular & Rubor facial, dispnéia, náuseas & 5 & 10 & Sim \\
\hline 6 & AIJ poliarticular & Dor torácica, rash, angioedema & 5 & 2 & Sim \\
\hline 7 & AIJ sistêmica & Dor torácica, dispnéia & 6 & 7 & Não \\
\hline 8 & AIJ sistêmica & Anafilaxia & 3 & 2 & Sim \\
\hline 9 & AIJ sistêmica & Náuseas & 3 & 4 & Não \\
\hline 10 & AIJ sistêmica & Rubor facial, rash, dispnéia, vômitos & 5 & 6 & Sim \\
\hline 11 & AIJ oligo/uveíte & Urticária/dispnéia & 3 & 1 & Sim \\
\hline 12 & AIJ poliarticular & Dor torácica, angioedema, rubor facial & 5 & 2 & Sim \\
\hline 13 & DII & Hipotensão & 5 & NR & Não \\
\hline 14 & AIJ sistêmica & Taquicardia, náuseas & 5 & 6 & Não \\
\hline 15 & Artrite psoriásica & Rash & 3 & 3 & Sim \\
\hline 16 & AIJ sistêmica & Rubor facial, rash, dispnéia, náuseas & 6,2 & 21 & Sim \\
\hline 17 & AIJ sistêmica & Dispnéia, hipertensão & 3 & 8 & Sim \\
\hline 18 & AIJ poliarticular & Taquicardia, rash & 4,2 & 3 & Não \\
\hline 19 & DMJ & Taquicardia, náuseas & 5,8 & 4 & Não \\
\hline 20 & DMJ & Angioedema, urticária & NR & $1-3$ & Sim \\
\hline 21 & AIJ poliarticular & Dispnéia, cianose & 5,5 & 3 & Sim \\
\hline 22 & AIJ oligo/uveíte & Rubor facial, rash & 4,6 & 3 & Não \\
\hline 23 & Policondrite & Taquicardia/náusea & 5,7 & 11 & Não \\
\hline 24 & Policondrite & Anafilaxia, rash, dispnéia, náuseas & NR & 10 & Sim \\
\hline 25 & ARE & Anafilaxia, dispnéia, angioedema & NR & 10 & Sim \\
\hline
\end{tabular}

AIJ = artrite idiopática juvenil; oligo $=$ oligoarticular; $\mathrm{EAJ}=$ espondiloartropatia juvenil; $\mathrm{TRAPS}=$ síndrome periódica associada ao receptor de TNF; DII $=$ doença inflamatória intestinal; $\mathrm{DMJ}=$ dermatomiosite juvenil; $\mathrm{ARE}=$ artrite relacionada à entesite; $\mathrm{NR}=$ não referido. 
Dos 58 pacientes analisados neste estudo, $25(43,1 \%)$ apresentaram efeitos adversos durante a infusão e, em 17 $(29,3 \%)$, a medicação teve de ser suspensa. Os resultados do presente estudo estão de acordo com trabalhos da literatura que relatam que reações agudas durante a infusão de infliximabe são comuns ${ }^{(7,10,12)}$. Kolho, Ruuska e Savilahti ${ }^{(10)}$ observaram $35 \%$ de reações durante a infusão de infliximabe em 23 crianças, a maior parte abaixo dos 10 anos, e 50\% destas reações foram anafilaxia ou urticária grave. Gerloni et $a l^{(7)}$ avaliaram 24 pacientes, sendo 19 adultos jovens e cinco crianças, e encontraram efeitos adversos em $50 \%$. Em um grupo de 56 pacientes, Pontikaki et al. ${ }^{(12)}$ encontraram efeitos adversos em metade dos casos, e em mais de um terço, a medicação teve de ser suspensa.

As reações observadas no estudo em análise são as mesmas descritas nestes trabalhos e relacionadas à hipersensibilidade: náuseas e vômitos, dispnéia e sensação de falta de ar, choque anafilático, angioedema, rubor facial, além de urticária, hipertensão, hipotensão, tosse, dor torácica, sensação de morte, taquicardia e cianose $\mathrm{e}^{(7,10,12)}$.

No presente estudo, as reações adversas foram mais freqüentes entre a quarta e a sexta aplicação, embora em um caso tenha ocorrido na primeira e, em outro, na décima oitava. Estudos na literatura observaram maior freqüência de reações alérgicas nas primeiras quatro infusões ${ }^{(9,13)}$.

O infliximabe é um anticorpo quimérico e inclui antígenos murinos, o que favorece a formação de anticorpos antiinfliximabe. Miele et al. ${ }^{(14)}$ observaram que mais de um terço de seus pacientes desenvolveram anticorpos durante

\section{REFERÊNCIAS}

1. Culy CR, Keating GM: Etanercept, an updated review of its use in rheumatoid arthritis, psoriatic arthritis and juvenile rheumatoid arthritis. Drugs 62: 2493-537, 2002.

2. Asadullah K, Docke WD, Volk HD, Sterry W: The pathophysiological role of cytokines in psoriasis. Drugs Today 12: 923-4, 1999.

3. Ardizzone S, Porro GB: Inflammatory bowel disease: new insights into pathogenesis and treatment. J Intern Med 252: 475-96, 2002.

4. Hashkes PJ, Laxer RM: Medical treatment of Juvenile Idiopathic Arthritis. JAMA 29: 1671-84, 2005.

5. Lovell DJ, Giannini EH, Reiff A, et al.: Pediatric Rheumatology Collaborative Study Group. Long-term efficacy and safety of etanercept in children with polyarticular-course juvenile rheumatoid arthritis: interim results from an ongoing multicenter, open-label, extended-treatment trial. Arthritis Rheum 48: 218-26, 2003.

6. Lahdenne P, Vahasalo P, Honkanen V: Infliximab or etanercept in the treatment of children with refractory juvenile idiopathic arthritis: an open label study. Ann Rheum Dis 62: 245-7, 2003. infusões repetidas. Reações infusionais são consideradas secundárias à formação destes anticorpos ${ }^{(15)}$.

Chama a atenção o fato de todos os pacientes do presente estudo terem sido medicados previamente à infusão com infliximabe, com o objetivo de prevenir reações de hipersensibilidade, entretanto, de acordo com os resultados encontrados, em alguns casos elas não foram evitadas.

Recentemente, Ruperto et al. ${ }^{(16)}$, em um estudo multicêntrico com 122 crianças observaram reações relacionadas à infusão em um grande número de pacientes que utilizaram a dose de $3 \mathrm{mg} / \mathrm{kg}$ (35\%) e em 17,5\% daqueles que utilizaram doses iguais ou maiores a $6 \mathrm{mg} / \mathrm{kg}$ e em $8,3 \%$ do grupo placebo. As reações graves também foram mais freqüentes no grupo que usou dose menor. Os autores concluem que o perfil de segurança do infliximabe na dose de $3 \mathrm{mg} / \mathrm{kg}$ parece ser menos favorável do que na dose de $6 \mathrm{mg} / \mathrm{kg}$, com incidência duas vezes maior de reações infusionais (freqüentemente graves), maior incidência de anticorpos contra infliximabe e maior freqüência de formação de anticorpos antinúcleo e anti-DNA. Entretanto, deve-se considerar que neste estudo os pacientes que receberam a dose de $3 \mathrm{mg} / \mathrm{kg}$ utilizaram o infliximabe por tempo mais prolongado, o que pode também ter contribuído para a ocorrência dos efeitos adversos.

Em resumo, a freqüência e a gravidade dos efeitos adversos do infliximabe devem ser consideradas, não apenas por ocasião da sua indicação, mas principalmente por ocasião da escolha do local para a sua aplicação, que deve contar com estrutura para atendimento de urgência e pessoal habilitado.

7. Gerloni V, Pontikaki I, Gattinara M, et al.: Efficacy of repeated intravenous infusions of an anti-tumor necrosis factor $\alpha$ monoclonal antibody, infliximab, in persistently active, refractory juvenile idiopathic arthritis. Arthritis Rheum 52: 548-53, 2005.

8. Foeldvari I, Nielsen S, Kummerle-Deschner J, et al.: Tumor necrosis factor-alpha blocker in treatment of juvenile idiopathic arthritis-associated uveitis refractory to second-line agents: result of a multinational survey. J Rheumatol 34: 1146-50, 2007.

9. Crandall WV, Mackner LM: Infusion reactions to infliximab in children and adolescents: frequency, outcome and a predictive model. Aliment Pharmacol Ther 17:75-84, 2003.

10. Kolho K-L, Ruuska T, Savilahti E: Severe adverse reactions to infliximab therapy are common in young children with inflammatory bowel disease. Acta Paediatrica 96: 128-34, 2007.

11. de Oliveira SK, de Almeida RG, Fonseca AR, et al.: Indications and adverse events with the use of anti-TNF alpha agents in pediatric rheumatology experience of a single center. Acta Rheumatol Port 32: 139-50, 2007. 
12. Pontikaki I, Gerloni V, Gattinara M, et al.: Effetti collaterali del trattamento com inibitori del TNF $\alpha$ nell'artrite idiopatica giovanile. Reumatismo 58: 31-8, 2006.

13. Jacobstein DA, Markowitz JE, Kirschner BS, et al.: Premedication and infusion reactions with infliximab: results from a pediatric inflammatory bowel disease consortium. Inflamm Bowel Dis 11: 442-6, 2005.

14. Miele E, Markowitz JE, Mamula P, Baldassano RN: Human antichimeric antibody in children and young adults with inflammatory bowel disease receiving infliximab. J Pediatr Gastroenterol Nutr 38: 502-8, 2004.

15. Baert F, Noman $M$, Vermeire $S$, et al.: Influence of immunogenicity on the long-term efficacy of infliximab in Crohn's disease. N Engl J Med 348: 601-8, 2003.

16. Ruperto N, Lovell DJ, Cuttica R, et al.: A randomized, placebocontroled trial of infliximab plus methotrexate for the treatment of polyarticular-course juvenile rheumatoid arthritis. Arthritis Rheum 56: 3096-106, 2007. 